\title{
Ueber Empis ciliata Fbr. und über die ihr zunächst verwandten Arten
}

vom

Director H, Loew in Meseritz.

U nter denjenigen Empis-Arten, welche ganz und gar sehwarz sind, eben so gefärbte Schwinger haben und bei denen die Augen des Männchens zusammenstofsen, befinden sich eino Anzahl, welche sich von allen übrigen Arten durch ihre erheblichere Gröfse und durch die viel ausgebreitetere und dichtere Behaarung des Thoraxrückens auszeichnen; in der Nähe seines Seitenrandes steben, wie bei den anderen Arten, starke Borsten, die übrige Behaarung ist. weniger borstenartig als bei jenen, zuweilen ziemlich zart; die Mittelstrieme ist stets halbirt, aber nicht, wie bei den meisten jener Arten, durch eine schmale Linie, sondern durch einen verhältaifsmäfsig breiten Zwischenraum, so dafs die beiden Tbeile der Mittelstrieme stets weit, oft ganz auffallend weit ron einander entfernt liegen; diestben sind in der Regel unbehaart, oder doch mit viel sparsamerer Behaarung besetzt, während sich die Behaarung sonst ubber den ganzen Thoraxrücken gleichmälsig ausbreitet. Diese Arten bilden einen eigenen Verwandtschaftskreis, welchen ich nach der bekanntesten dieser Arten denjenigen der Empis ciliata Fabr. nenven will; die andern Arten gehören fast ohne Ausnahme in den Verwandtschaftskreis der Empis chioptera Fall.

In den Verwanditschaftskreis der Emp. ciliata gehören von den bisher beschriebenen Arten, aufser $\boldsymbol{E} m \boldsymbol{m}$. ciliat a selbst, nur noch Emp. nigerrima Lw. und Emp. hirta Lw.; aufserdem besitze ich drei unbescbriebene, in denselben zu stellende Arten, welche ich Empis pilicornis, fumosa und nigricoma nennen will.

Ich will versuchen diese sechs Arten etwas genauer auseinander zu setzen. 


$$
2
$$


1. Empis ciliata ist an folgenden Merkmalen leicht zu erkennen. Körper glänzend schwarz; Thoraxrïcken ziemlich matt. doch mit glänzend schwarzen Striemen, welche sämmtlich behaart sind. Die Behaarung des Körpers schwarz, bei dem Männchen viel länger als bei dem Weibchen, auf dem Thoraxräcken, besonders bei verflogenen oder lange in der Sammlung aufbewahrten Exemplaren rufsbräunlich, an den Vorderhüften und an der Basis des Finterleibs weifslich, doch auf dem ersten Abschuitle des lelztern zum grofsen Theile schwarz; dem schwarzen Haarschirme vor den Schwingern sind ebenfalls ziemlich viele weifse Haare beigemengt. Die Flügel sind bei ganz frischen Exemplaren ziemlich intensiv schwärzlich mit schwarzem Vorderrandssaume und Randmale, bei verflogenen oder veralteien mehr rufsfarben mit braunschwaizem Vorderrandssaume und Randmale; an der Basis derselben zeigt sich nicht die geringste Spur von gelberer Färbung; die dritte Längsader jst an ihrem alleräufsersten Ende el was vorwärts gebogen; Discoidalzelle verhältuifsmäfsig klein, am Ende sebr schief. Beine des Männchens kurzborslig; Mittel- und Hinlerschenkel des Weib. chens auf der Oberseite von kürzeren, auf der Unterseile von längeren, sehr schmalen und spitzigen Schuppen gefiedert, ebenso die Unterseite der Hinterschienen; die Unlerseite der Mittelschienen desselben trägt nur in der Nähe der Basis schmale Schuppenhärchen. Das schwarze, mit dichter schwarzer Behaarung besetzte Hypopygium des Männchens ist von ungewöhnlichem Bauc, indem es zum grofsen Theile unterhalb des Hinterleibs liegt, wo es bereits an dev Basis des vorletzten Abscbnitts beginot; die seitlichen Lamellen desselben reichen erheblich über das Fnde des lełzten Hinterleibsabschnitts hinaus, sind am Ende ziemlich abgerundet, obèn gerade abgeschnitten und mit einem schmalen kastanienbraunen Rande versehen; die obere lamelle ist verhältnifsmäfsig ziemlich grofs und bat in der Mitte ibres Oberrandes zwei schmale, zipfelförmige Anbänge; der gelbe, nicht sehr starke Penis liegt unterhalb des Hypopygiums und lăuft in einem sehr schwach gekrimmten Bogen von der Basis bis zur Spitze desselben.

2. Empis nigerrima ist nur wonig kleiner, aber erheblich schlankbeiniger als Emp. ciliata. Sic unlerscheidet sich von den anderen Arten schon durch ihre völlig glasartigen Flügel mit schwarzbraunen Adern und schwarzem Randmale auf das Bestimmteste und Leichteste. Sic ist glänzend schwarz, doch die Oberseite des Thorax vicl matter and die Striemen derselben nur schwach 
gleifsend, so dafs man dieselben nur mit Mühe wahrnimmt. Die Behaarnng ist schwarz, bei dem Männchen lănger als bei dem Weibchen, doch nicht ganz so lang wie bei dem Männchen der Emp. ciliata; auf der Oberseite des Thorax und an den Vorderhüften hat sie oft eine hellere, mehr rufsbrăunliche Fărbung; der Thoraxrücken erscheint bei nicht sehr genauer Untersuchung von derselben gleichmärsig bedeckt, dach findet man bei grölserer Aufmerksamkeit zwei schmale, unbehaarte Längsstreifen, welche in der That weiter nichts sind, als die beiden, durch einen ganz auffallend breiten, dichtbehaarten $Z$ wischenraum getrennten Theile der Mittelstrieme; vor den Scbwingern stehen keine anderen als schwarze Hare, dagegen sind die vorderen Hinterleibsringe an den Seiten und der gröfste Theil des Bauchs weifslich behaart. Die Beine sind verbältnifsmäfsig ziemlich schlank, die Füfse gegen das Ende bin besonders dünn; die Beine des Männchens sind mit zerstreuten, mäfsig langen Borsten besetzt; Hinterschenkel and Hintersebienen des Weibchens sind beiderseils von ziemlich kurzen, kaum deutlich plattgedrückten Här-. chen sehr dicht gewimpert, doch sind diese Härchen auf der Oberseite der Hinterschienen deutlicher plattgedrückt und etwas länger. Das Flügelgeäder äbnelt dem der Emp. ciliata, indem das äufserste Ende der dritten Längsader etwas vorwärts gebogen, die Discoidal. zelle aber verhältnifsmälsig klein und an ihrem Ende schief abgeschnitten ist. Das Männchen zeichnet sich noch dadurch aus, dafs ein grofser Theil des vorletzten und der selir kurze letzte Hinterleibsabschuitt mit brauner Bestäubung bedeckt sind. Das schwarze und schwarzbehaarte Hypopygium ist ziemlich klein, rundlich, vollkommen geschlossen, so dafs vom Penis nichts zu sehen ist; die untere, stark gewölbte Seite desselben bildet der ungewöhnlich stark entwickelte achte Bauchabschnitt, welcher an jeder Seite mit einem warzenartigen Höcker besetzt ist; die seitlichen Lamellen sind klein und sehr stumpf; die obere Lamelle ist in zwei länglich - eiförmige, ziemlich mattschwarz gefärbte Lappen getheilt, welche die obere Oeffnung des Hypopygium von der Seite her mehr oder weniger überdecken.

3. Empis pilicornis ist ebenfalls schwarz, doch mit sehr dünner und deshalb wenig in die Augen fallender Bestäubung, also nur wenig glänzend und nicht tiefschwarz. Der Thorax zeigt kaum eine Spur von dunkler Striemung, dagegen zeigen die einzelnen Hinterleibsabschnitte bei von hinten cinfallender Beleuchtung jeder einen schmalen, aber scharfbegrenzten und sehr deutlichen weifsen 
Hinterrandssaum. Die Behaarung des ganzen Körpers ist obne alle Ausnahme schwarz. Das erste Fühlerglied ist linger und dicker als bei den anderen Arten und mit ganz auffallend langer, aber keineswegs borstenartiger schwarzer Behaarung beselzt; auch das gerundete z.weite Fühlerglied ist erbeblich länger als bei den andern Arten behaart. Die Oberseite des Thorax sclseint überall gleichnaälsig behaart zu sein, doch finden sich in der That zwei ganz schmale, kahle Streifen, welche den weit von einander entfernt liegenden beiden Theilen der Mittelstrieme entsprechen. Das Hypopygium ist kaum von mittlerer Gröfse und von der Seite her zusammengedrückt; vor demselben trelen der siebente und achte Bauchabschnitt kappenförmig vor; die seitlichen Lamellen sind von sehr einfacher Form mit geradem Oberrande und gebogenem Unterrande; die obere Lamelle ist in zwei dreieckige Zipfel getheilt, welche dieselbe Lage gegeneinander haben, wie die Seitenlamellen; vom Penis ist nichts zu sehen. Die Beine sind schlank, besonders die hintersten; auch die Füfse sind dünn, das erste Glied derselben durchaus nicht stärker als die folgenden; mit Ausnabme der Vorderund Hinterschenkel sind die Beine mit abstehenden, recht ansehnlichen Borsten besetzt. Flügel verhältnifsmäfsig lang, rauchschwärzlich, an der Basis undeutlich lehmfarbig; das lange Randmal braun. schwarz, das äulserste Ende der dritten Längsader etwas vorwärtsgebeugt; Discoidalzelle nicht grofs, am Ende schief. - Das Weibchen kenne ich nicht.

4. Empis hirta ist schwarz, mit deutlicher bräunlichgrauer, auf dem Hinterleibe mebr brauner Bestäubung, deshalb etwas grauschwarz und wenig glänzend. Die Behaarung des Körpers ist ohne Ausnahme schwarz, doch hat sie bei manchen Exemplaren auf dem Thoraxrücken ein auffallend fuchsiges Ansehen; bei dem Männchen ist sie länger als bei dem Weibchen, aber kürzer als bei den vorhergehenden Arten. Das erste Fühlerglied ist weder verlängert noch verdickt, auch die Behaarung desselben verbältnifsmäfsig nicht länger, als bei Emı. ciliata und nigerrima. Die Oberseite des Thorax zeigt nur eine sehr Bchwache Spur von Glanz; von hinten her betrachtet erscheinen die Inlerstitien schwärzer als die Striemen; die beiden Theile der Mittelstrieme sind ziemlich schmal und unbehaart wie bei den beiden zunăchst vorhergehenden Arten, aber weniger weit von einander entfernt. Die braune Bestäubung des Hinterleibs nimmt man nur dann deutlich wahr, wenn man die Flyche desselben in ganz schräger Richtung betrachtet. Das schwarze und 
mit schwarzer Behaarung beselzte Hypopygium ist klein; es beginnt auf der Unterseite des Hinterleibs schon auf der Mitte des vorletzten Abschnilts; die seitlichen Lamellen desselben sind klein; die obere Lamelle ist in zwei nebeneinander liegende, an ihrer Basis breitere Zipfel getheilt, deren sehr spitzes Ende sich abwärts beugt; am Hinterrande des letaten Bauchabschnitts befindet sich eine längliche, mäfsig breite, auf ihrer Unterseite gewölbte und an ihrem Ende abgestutzte, glänzend pechbraune Platte, über welcher der gebogene, scbmutziggelbe Penis entspringt, welcher leicht übersehen werden kann, da er sich dem Contoure des Hypopygiums ziemlich genau anschliefst. Die Beine sind ziemlich lang, besonders die hintersten; die Mittelschienen sind mit einer mäfsigen Anzahl nicht sehr langer Borsten besetzt; die Hinterschienen und das erste Glied der Hinterfüfse sind bei dem Mănnchen von kräftigem Baue, erstere auf der Oberseite dicht mit langen, borstenartigen Haaren besetzl, letzteres auf der Oberseite mit borstenartigen Haaren auf der Unterseite mit zahlreichen Stachelborsten; bei dem Weibchen ist die Bebaarung der Linteren Schenkel gröber als bei dem Männchen, doch sind die eiuzelnen Haare vicht plattgedrückt, so dafs die Schenkel nur wimperartig behaart erscheinen; dasselbe gilt von der Behaarung seiner Hinterschienen, doch erscheint diesclhe nur an der Basis der Unterseite etwas wimperartig, wäbrend sie auf der Oberseite dazu viel zu lang und ungleich ist. Die Flägel sind schwärz. lich, bei veralteten Exemplaren melor braunschwärzlich, an der Basis in ziemlicher Ausdehnung zwar nicht gerade auffallend, aber doch recht deutlich lehmgelblich; das lange Randmal ist braunschwař; das äufserste Eode der dritten Längsader zeigt keine Spur von Vorwårtsbeugung; die Discoidalzelle ist von mittlerer Grölse, namentlich gröfser als bei den vorhergehenden Arten und am Ende viel gerader abgeschnitten.

5. Empis fumosa, von der ich nur ein einzelnes Mănnchen besitze, ist der Emp. hirta so ähnlich, dafs sie aus der Beschreibung jener mit Sicherheit erkannt werden kann, wenn man auf folgende Unterschiede achtet. Die Flügel sind im Verhältnisse zux Körpergrölse länger und breiter und nur rufsbräunlich gefärbt, also viel lieller; das Randmal ist braun; die Flügelwurzel zeigt kaum eine Spur von mehr in das Gelbe ziehender Färbung. Die Bestäubung auf dem Thoraxrücken ist viel dünner und von dunklerer Färbung der Interstitien ist nichts wahrzunehmen. Die Hinterschen. kel sind merklich kräfliger und efwas kürzer, die Hinterschienen 
breiler und die borstemartige Behaarung ihrer Oberseite weitläufiger; das erste und alle folgenden Glieder der Hinterfüfse etwas stärker. Das Hypopygium läfst leider keine genaue Untersuchung zu, doch ist wohl zu sehen, dafs seine Bildung derjenigen, welche es bei Emp. hirla hat, äufserst ähnlich ist. - Das beschriebene Exemplar ist angeblich in Ungarn gefangen worden.

Noch mufs ich schliefslich bemerken, daf's das Weibchen der Pachymeria lugubris $m$. leicht für das einer in gegenwărtigen Verwandtschaftskreis gebörigen Ari gehalten werden kann; die stärkere Entwickelung der Hinterscbenkel und vor allen die breite Stirn des Männchens lassen ihre Unterbringung in der Gattung Pachymeria als die naturgemälsere erscheinen.

6. Von Empis nigriroma fing ich im Juli auf dem Wiener Schneeberge zwei Männchen, zu gleicher Zeit auch ein Weibchen, welches wahrseheinlich zu denselben gehört. - Das Mänuchen, auf welches allein ich für jetzt den gewählten Namen anwende, gleicht dem Männchen der Emp. fumosa onf das täuschendste, ist aber soviel kleiner, dafs es nicht für eine Abänderung derselben gehalten werden kann. Sonst sehe ich nur folgende Unterschiede: Die Behaarung des Thoraxrückens ist verhältnifsmäfsig etwas länger; die Behaarung der Vorderhüften ist sebr viel weniger dicht und die Behaarung auf der Oberseite der Vorderschienen verhältnifsmäfsig etwas stärker und verhâltnifsmäfsig auch etwas länger; die Färbung der Flügelflăche zieht ein wenig mehr in das Schwärzlichgraue.

Das Weibchen, welches ich mit den beschriebenen Männchen zugleich fing, hat leider die Schwinger vertoren, so dafs ich jetzt weniger fest überzeugt bin, in ihn das Weibchen der Emp. nigricoma vor mir zu haben, als ich es bei dem Fange desselben war. Die deutliche Behaarung der Seitenstriemen des Thorax macht es sehr wahrscheinlich, dafs dasselbe wenigstens einer Art gegenwärtiger Gruppe angehöre. Die Körperfărbung wie bei Emp. nigricoma $\delta$, doch der Hinterleib glänzender. Die Behaarung überall ohne Ausnahme schwarz, viel kürzer als bei Emp. nigricoma $\delta$. Flügel viel dunkler, braunschwärzlich, elwa wie bei Emp. pilicornis und hirta, so dafs die mehr lehmgelbliche Farbe der Flügelwurzel etwas deullicher gegen die Färbung der äbrigen Flügelfẳ. che contrastirt. Die Vorderschenkel haben auf der Oberseite wimperartige, in der Nähe ihrer Spitze besonders dichte Behaarung; Vorderschienen auf der Oberseite kurz gefiedert. nur ganz an der 
Wurzel wimperartig behaart, auf der Unterseite mit sehr kurzer, wimperartiger Behaarung; erstes Glied der Vorderfüfse auf der Oberseite nil anliegender, leicht zu übersehender Bewimperung. Miltel- und Hinterschenkel beiderseits lang gefiedert; die Mittelschienen baben auf der Oberseite kürzele und mehr anliegende Befiederung, welche an dem Ende derselben zuletzt sehr kurz wird; auf der Unterseite derselben zéigt sich nur in der Nähe der Basis eine wimperartige Befiederung, welche sehr bald in eine kurze, gewöhnliche Behaarung von wimperartiger Beschaffenheit übergelıt; die Hinterschienen haben auf der Oberseite ziemlich lange Befiederung, welche gegen das Ende hin kürzer wird und schon etwas vor demselben in gewöhnliche Behaarung äbergeht; die erste Hälfle ihrer Unterseite ist ebenfalls ziemlich lang gefiedert, doch wird dicse Befiederung schon gegen die Mitte derselben hin wieder ziemlich kurz, geht hier in Bewimperung und sehr bald in wimperartige Behaarung über. Das erste Glied der Mittel- und Hinterfüfse ist auf der

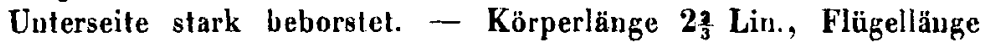
$2 \frac{1}{3}-2_{\frac{5}{2}}$ Lin.

Die Diagnosen der besprochenen sechs Empis-Arten können etwa in folgender Weise aufgestellt werden.

1. Empis ciliata Fabr. $\delta$ el + . Atra, nitida, thoracis ta. men dorso praeter vittas subopaco, nigro-pilosa, coxis anticis abdominisque basi allido-pilosis, alis nigricantibus, limbo costali nigro, basi non lutescente. Hypopygium maris longiusculum, filamento centrali exserlo infero, lamellâ superâ appendicibus duabus instruct $\hat{\alpha}$. Foeminae tibiae posticae infra, femora posteriora ulringue pennata.

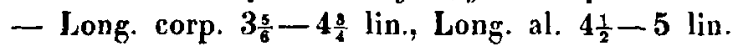

Syn. Empis ciliata Fabr. Ent. Syst. IV. 405. 9.

Empis pennipes Meig. Klass. I. 219. "2.

Empis pennata Panz. Faun. XCI. 22.

Empis boja Schrank Faun. boic. III. 2572.

Empis ciliata Meig. Syst. Beschr. III. 20. 7.

Empis ciliata Macq. Dipt. d. N. Empid. 122. 10.

Hab. Europa media.

2. Empis nigerrima Lw. ठౌ et ․ Atra nitida, dorso tr. men thoracis tolo subopaco, nigro pilosa, abdominis lateribus antice et ventre praeter apicem albido-pilosis, alis pure hyalinis, stigmate nigro. Hypopygium maris lreve, rolundatum, clausum, filamento centrali operto. Pedum posticorum tibiae et femora in foemina 
pilis vix compressis confertim sed breviter ciliata. - Long. corp. $3 \frac{3}{2}-4$ lin., Long. al. $4 \frac{1}{3}-4 \frac{1}{2}$ lin.

Syn. Empis nigerrima Loew Wien. ent. Monatschr. VI. 171. 49.

Hab. Varna, Schumla.

3. Empis pilicornis m. o'. Nigra, tenuissime cinereo-pollinosa, tota nigro-pilosa, primo antennarum articulo crasso et confertissime pilis perlongis nigris vestilo, pedibus tenuibus, selosis, alis nigricantibus prope basim subhutescentibus. Hypopygium parvum, compressum, clausum, filamento centrali operto. - Long. corp. 4 lin., Long. al. 4 lin.

Hab. Hispania.

4. Emp is hirla Lw. of et 9. Nigra, polline obscure cinereo aspersa, tola nigro-pilosa, tibiis posticis maris supra confertim nigro-pilosis, pedibus foeminae simplicibus, alis nigricantibus, basi lutescente, stigmate fusco-nigro. Hypopygium parvum, nigro-pilosum, lamellis lateralibus minutis, lamellâ superâ divisâ, filamento centrali lamella inferd suffullo. - Long. corp. $2 \frac{2}{3}-2 \frac{3}{4}$ lin., Long. al. $2 \frac{2}{3}$ lin.

Syn. Empis hirta Loew Berl. ent. Ztsehr. IX. 240. 12.

Hab. Imeretia.

5. Empis fumosa u. o'. Nigra, polline cinereo leviler aspersa, tota nigro-pilosa, coxis anticis confertim pilosis, tibiis pósticis latis, supra minus confertim nigro-pilosis, alis subfuscis, basi vix obsoletissime lutescende, stigmate fusco. Hypopygium parvum, nigro-pilosum, lamellis lateralibus minutis, lamellâa supr $\hat{a}$ divisd, filamento centrali lamella inferá suffulto. - Long. corp. $2 \frac{2}{3}$ lin., Long. al. 3 lin.

Hab. ?Hungaria.

6. Empis nigricoma m. ठ. Nigra, polline cinereo leviler aspersa, tota nigro-pilosa, coxis anticis modice pilosis, tibiis posticis latiusculis, supra minus confertim nigro-pilosis, alis subfuscis, basi vix obsoletissime lutessente, stigmate fusco. Hypopygium parvum, nigro-pilosum, lamellis lateralibus minutis, lamellâ superä divisa, filamento centrali lamellâ inferâ suffulto. - Long. corp. 2 lin., Long. al. $2 \frac{1}{4}$ lin.

Hab. Austria. 
Eine in Mai in ganz Deutschland häufige Art, welche ich $\mathbf{E m}$ pis pilosa nenne, steht hinsichtlich der Behaarung des Thoraxrückens den Arten aus dem Verwandtschaftskreise der Emp. ciliala Fabr. so nahe, dafs sic, obgleich ihre Schwinger hell gefärbt sind, demselben füglich beigezählt werden kano. Ich will deshalb die Beschreibung derselben hier folgen lassen.

Empis pilosa m. ठ et + . Nigra, polline cinereo leviter. aspersa, totu nigro-pilosa, pedibus utriusque sexus simplicibus, alis ex fusco nigricantibus, sligmate fusco, halleribus luteis. Hypopygium maris parvum, nigro-pilosum, lamellis laleralibus minutis, lamellá super $\hat{a}$ divisâ, flamento centrali lamellâ inferâ suffulto. - Long. corp. $2 \frac{1}{\frac{1}{2}}-2 \frac{1}{3}$ lin., Long. al. $2 \frac{1}{4}-2 \frac{5}{\frac{5}{2}}$ lin.

Hab. Germania.

Schwarz, mit dünner, grauer Bestäubung, welche an den Brustseiten und am Bauche am deutlichsten ist und dem Thoraxiücken ein grauschwarzes Ansehen giebt; betrachtet man letzteren von vorn her, so erscheinen die beiden Theile der Mittelstrieme heller grau als seine übrige Fläche, wăhreod, wenn man ihn von hinten her ansieht, die Interstitien eine sehr auffallende schwarze Färbung zeigen. Auf dem gleifsend schwarzen Hinlerleibe des Männchens bemerkt man die graue Bestäubung nur scbwer, auf dem ziemlich glänzenden Hinterleibe des Weibchens so gut wie gar nicht. Die Behaarung des ganzen Körpers ist ohne alle Ausnahme schwarz, bei dem Männchen von ziemlicher Länge, bei dem Weibchen viel kürzer. Das dritte Fühlerglied ist verhältnifsmäfsig etwas kurz und an seiner Basis breiter als gewöhnlich. Flügel braunschwärzlich getrübt, etwa von der Färbung wie bei Emp. pilicornis und hirta; die Aderı und das sehr deutliche Randmal dunkelbraun.

Die Beine des Männchens sind einfach; die Behaarung derselben ist märsig dicht; Oberseite der Vorderschienen mit ziemlich langer Behaarung; die borstenartigen Haare auf der Unterseite der Mittelschenkel nicht sehr zahlreich, in der Nähe der Basis nicht zusammengedrängt, nur auf der Wurzelhälfte der Schenkel von ansehnlicher Lănge, weiterhin immer kürzer; Mittelschienen verhältnifsmäfsig nur sparsam mit borstenartigen Haaren besetat; die an der Spize derselben befindlichen borstenartigen Haare nicht lang; Unterseite der Hintersehenkel nur nit sehr märsic langen Haareu; Hinterschienen auf der Oberseite in der gewöhnlichen Weise mit ungleich langer, ziemlich borstenartiger Behaarung, welche nicht sehr dicht ist; auch das ersle Glied der Hinlerfüfse ist auf der Ober- 
seite in ähnlicher Weise behaart, auf der Unterseite aber ziemlich stark bedornt, Hypopygium klein, schwarz behaart; die seitlichen Lamellen sehr kurz und ziemlich stumpf, die wenig deulliche obere Lamelle gespalten; der Penis ist entweder ganz eingeschlossen, oder man nimmt doch nur seinen unleren, steil aufsteigenden Anfang wahr; er ist von einer kurzen schwarıen Lamelle, welche am Hinterrande des letzten Bauchabschuitts entspringl, unterstätzt.

Die Beine des Weibchens sind durchaus einfach, ohne jede Spur von Befiederung oder Bewimperung; die Bebaarung derselben ist äufserst viel kürzer als bei dem Mănnchen, namentlich ist auch die Behaarung auf der Unterseite aller Schenkel nur kurz; an den Schienen ist sie etwas weniger kurz und ähnelt, von der länge alsgesehen, der des Männcheus.

Die in Sicilien einheimische Emp. macropalp a Egg., welche cbenfalls auf dem Thoraxrücken stark behaart ist, hat mit den hier besprochenen Arten keine nälsere Verwandischaft, sondern stelıt der bekannten Emp. livida Linn. am nächsten; wie bei dieser sind auch bei ihr die Flügel des Weibchens erheblich heller als die des $\delta$ und die Schaltader ist vor dem Flügelrande abgebrochen. Die Egger'sche Beschreibung des Männchens macht dieses zur Genüge kenntlich. Zur Beschreilung des Weibehens habe ich zu bemerken, dafs die Flügel desselben fast glasartig, gegen den Hinterrand und besonders gegen die Spitze hin aber grau sind; der ziemlich glänzende, braunschwarze Hinterleib desselben ist an den Seiten in ansebnlicher Ausdehnung, auf dem ganzen ersten Abschnitte und am Hinterrande der folgenden Abschnitte aschgraulich und matt. 